\title{
PROLIFERATION INDEX AS A PROGNOSTIC MARKER IN HEMANGIOPERICYTOMA OF THE HEAD AND NECK
}

\author{
Paul J. Kowalski, MD, Augusto F. G. Paulino, MD \\ Department of Pathology, University of Michigan Hospitals, Room 2G332, 1500 East Medical Center Drive, \\ Ann Arbor, Michigan, 48109-0054
}

Accepted 5 December 2000

\begin{abstract}
Background. Hemangiopericytoma (HPC) of the head and neck is a rare neoplasm whose biologic behavior is difficult to predict by means of conventional histologic parameters.

Methods. H \& E-stained sections from 12 cases of HPC were reviewed. Proliferation index was assessed using an immunoperoxidase stain for MIB-1 (Ki-67).

Results. The study group consisted of 4 adult men, 5 adult women, and 1 infant male. Necrosis, hypercellularity, and pleomorphism were found in 1, 5, and 6 case(s), respectively. The mitotic index per 10 high power fields varied from $0-1$ to 15 . Proliferation indices using MIB-1 ranged from $2.6 \%$ to $52.5 \%$. Clinical follow-up revealed 3 cases with recurrence all possessing proliferation indices of approximately $10 \%$.

Conclusions. Standard histomorphologic features may be inadequate predictors of clinical outcome. A proliferation index of $10 \%$ or greater may indicate a more aggressive subset of HPC of the head and neck. (C) 2001 John Wiley \& Sons, Inc. Head Neck 23: 492-496, 2001.
\end{abstract}

Keywords: head and neck; hemangiopericytoma; MIB-1 (Ki-67)

Hemangiopericytoma (HPC), first described by Stout and Murray ${ }^{1}$ in 1942 , is an uncommon vas-

Correspondence to: A. F. G. Paulino

Presented at the 5th International Conference on Head and Neck Cancer San Francisco, California, July 29, 2000

(c) 2001 John Wiley \& Sons, Inc. cular tumor that arises from pericytes. Although the precise function of pericytes is unknown, these ovoid cells are intimately associated around capillaries and possess structural similarity to smooth muscle cells and fibroblasts, suggesting a contractile and supportive role. ${ }^{2}$

Although these tumors represent only a small fraction of all head and neck tumors, approximately one fifth of all HPCs are seen at this site. ${ }^{3,4}$ HPCs more commonly arise in the lower extremity and pelvic retroperitoneum. ${ }^{5}$

The architectural pattern of HPC can be seen in other mesenchymal neoplasms. The diagnosis of HPC is one of exclusion and relies on the presence of characteristic histologic features. However, difficulties exist in attempting to predict biologic behavior based on conventional histopathologic parameters. The purpose of this study is to assess the histologic features and to evaluate the prognostic significance of the proliferation index in HPC of the head and neck.

\section{MATERIALS AND METHODS}

During the years of 1989 to 1999,12 cases (10 patients) of primary or recurrent HPC of the head and neck were retrieved from the surgical pathology files of the University of Michigan. The available clinical data were reviewed, and additional 


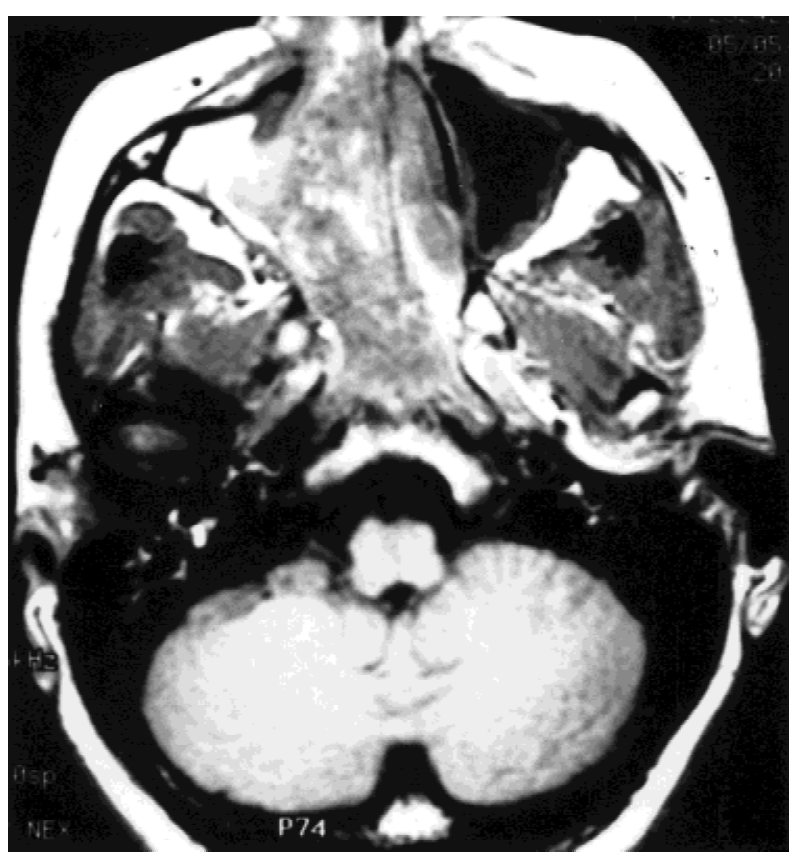

FIGURE 1. CT scan of a right nasal hemangiopericytoma infiltrating the nasal sinuses.

clinical information was obtained by contacting primary physicians. In this study group, there were 4 adult men (ages 25-83), 5 adult women (ages $45-55)$, and 1 infant male (2 months). Sites of involvement included the orbit $(n=4)$, maxillary sinus $(n=2)$, nasal cavity $(n=2)$, maxilla $(n=2)$ (Fig. 1), mandible $(n=1)$, and temporal region $(n=1)$. All patients were treated by surgical excision, and 1 patient received radiotherapy after 2 recurrences. The primary resec- tion was reviewed in 9 cases. One patient's material was a recurrence 20 years after treatment of a maxillary sinus HPC.

$\mathrm{H} \& \mathrm{E}$-stained sections from all cases were reviewed to confirm the diagnosis and to assess the following histologic features: pleomorphism, hypercellularity, necrosis, and the number of mitotic figures per 30 high power fields (hpf).

The proliferation index was determined as a percentage of cells, from a minimum of a 1000 counted, with positively staining nuclei. Formalin-fixed, paraffin-embedded tissue sections were stained immunohistochemically according to manufacturer's recommendations with MIB-1, a monoclonal antibody to a known recombinant peptide corresponding to the Ki-67 cDNA fragment (Immunotech, Marseilles, France, dilution: 1/25). The sections were pretreated with microwave antigen retrieval followed by staining with a standard avidin-biotinyl peroxidase complex method using an automated immunostainer (Ventana Medical Systems, Inc., Tucson, AZ). Immunoperoxidase staining for smooth muscle actin and S100 was also used on selected cases to exclude the possibility of other mesenchymal neoplasms.

\section{RESULTS}

The histopathologic features and clinical information of the 12 cases are summarized in Table 1. All cases microscopically consisted of branching vascular spaces with oval or spindle-shaped pericytes arranged in a random pattern filling the areas between the vessels (Fig. 2). Pleomorphism

\begin{tabular}{|c|c|c|c|c|c|c|c|c|c|}
\hline $\begin{array}{l}\text { Age }(y) / \\
\text { gender }\end{array}$ & Site & Pleomorphism & Necrosis & $\begin{array}{l}\text { Increased } \\
\text { cellularity }\end{array}$ & Mitosis* & $\mathrm{P} / \mathrm{R}+$ & PIf & $\begin{array}{l}\text { Follow- } \\
\text { up (mo) }\end{array}$ & Status $\S$ \\
\hline $55 / F$ & Orbit & No & No & No & $0-1$ & $\mathrm{P}$ & 3.9 & 105 & U \\
\hline $50 / F$ & Orbit & Yes & No & No & 15 & $P$ & 52.5 & 27 & NED \\
\hline $30 / \mathrm{M}$ & Orbit & No & No & Yes & $0-1$ & $\mathrm{P}$ & 9.4 & 15 & NED \\
\hline $49 / F$ & Nasal cavity & Yes & No & No & 0 & $P$ & 4.0 & 17 & NED \\
\hline $48 / F$ & Maxillary sinus & No & No & No & $0-1$ & $\mathrm{P}$ & 3.5 & 130 & U \\
\hline $27 / M$ & Temporal bone & Yes & No & Yes & $0-1$ & $P$ & 2.6 & 27 & NED \\
\hline $2 \mathrm{mo} / \mathrm{M}$ & Mandible & Yes & No & Yes & 4 & $P$ & 19.6 & 83 & NED \\
\hline $34 / F$ & Maxillary sinus & No & No & No & $0-1$ & $P$ & 3.6 & 136 & MR \\
\hline $42 / F$ & Maxilla & Yes & Yes & No & $0-1$ & $\mathrm{R}$ & 10.2 & 34 & MR \\
\hline $44 / F$ & Maxilla & Yes & No & Yes & $0-1$ & $\mathrm{R}$ & 16.8 & 13 & MR \\
\hline
\end{tabular}

*Mitoses/10 high power fields with 30 fields counted.

tLesion studied: primary $(P)$ or recurrence $(R)$.

$\neq$ Proliferation index percentage counting 1000 cells for MIB-1 nuclear positivity.

$\S M R=$ multiple recurrences; $N E D=$ no evidence of disease; $U=$ unknown. 


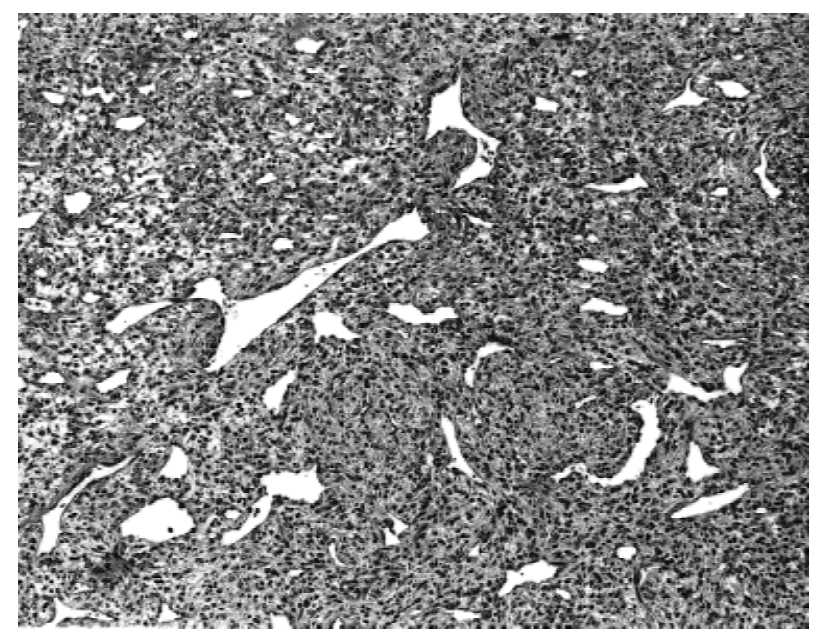

FIGURE 2. Branching vascular spaces with surrounding pericytes $(H \& E$, original magnification $\times 10)$.

and increased cellularity were observed in 6 and 5 cases, respectively, with 3 cases exhibiting both features. Focal necrosis was found in only 1 case. This patient subsequently had two recurrences, the first of which was pleomorphic with a low mitotic index, and the second was pleomorphic and hypercellular but lacked necrosis or an increased mitotic rate.

An increased mitotic index was observed in only 2 cases compared with the $0-1$ mitoses $/ 10 \mathrm{hpf}$ as seen in all other cases. The single congenital case involved a 2-month-old infant with more than 20 cutaneous lesions that spontaneously resolved after several months. This resection specimen was pleomorphic, hypercellular, and possessed an increased mitotic rate $(4 / 10 \mathrm{hpf})$ and proliferation index $(19.6 \%)$. Similarly, an orbital

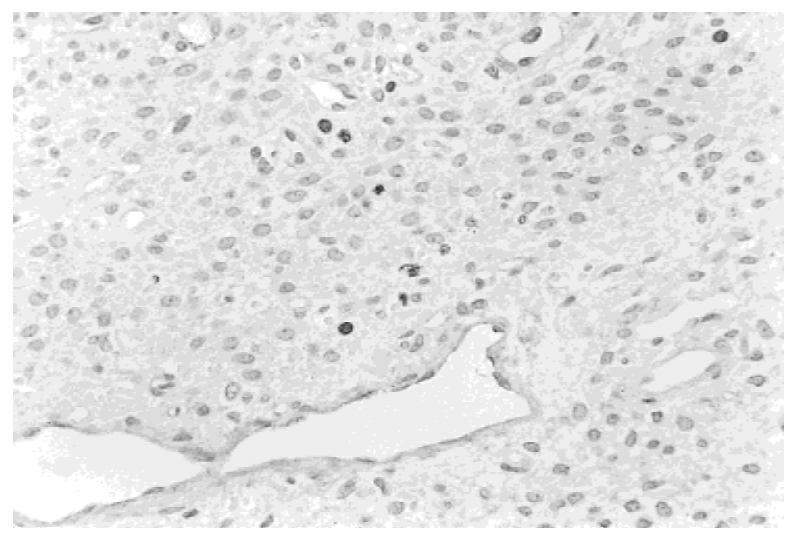

FIGURE 4. Positive nuclear staining for MIB-1 in an HPC of the temporal region, proliferation index $=2.6 \%(\mathrm{H} \& \mathrm{E}$, original magnification $\times 40$ ).

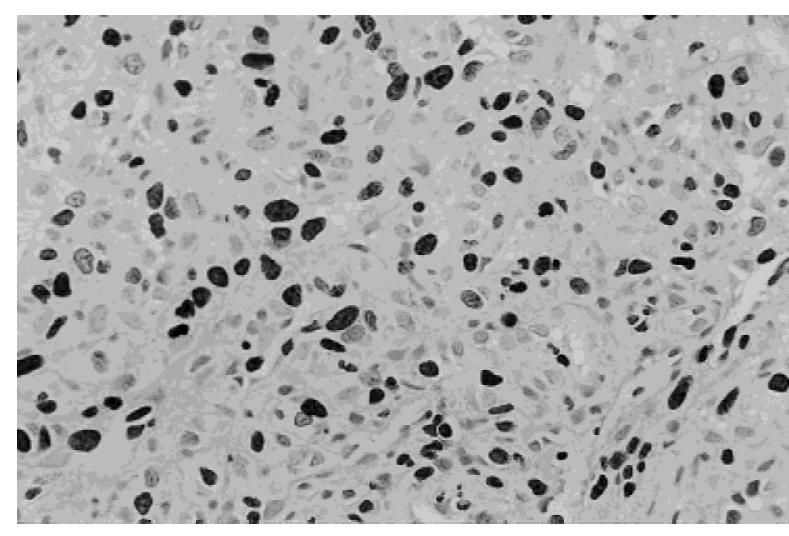

FIGURE 3. Positive nuclear staining for MIB-1 in an orbital lesion, proliferation index $=52.5 \%$ ( $\mathrm{H} \& \mathrm{E}$, original magnification $\times 40$ ).

lesion was the most mitotically active ( $15 / 10 \mathrm{hpf})$ and the most proliferative at $52.5 \%$ (Fig. 3).

The proliferation index ranged from $2.6 \%$ to $52.5 \%$. One case exhibiting both pleomorphism and increased cellularity possessed the lowest proliferation index at $2.6 \%$ (Fig. 4). The proliferation index of 6 cases, including the congenital case, was approximately 10\%. The 3 recurrent lesions had proliferation indices ranging from $9.8 \%$ to $16.8 \%$ (Fig. 5). In addition, two orbital lesions, with proliferation indices of $9.4 \%$ and $52.5 \%$, have not recurred after 15 and 27 months of follow-up.

\section{DISCUSSION}

Numerous studies have described the clinical and pathologic features of HPC since its first description in $1942 .{ }^{1}$ The lesion predominates in the retroperitoneum and lower extremities, with the head and neck as the third most common location. ${ }^{3,6,7}$ HPC can occur in any age group, but the

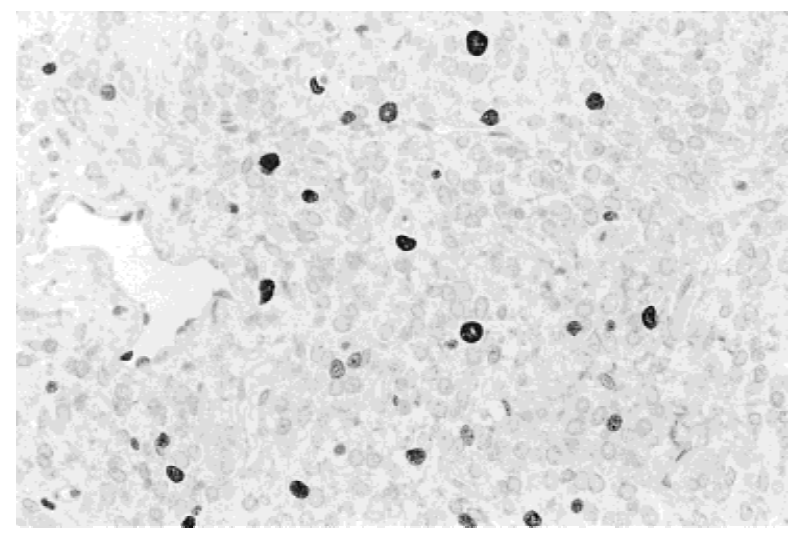

FIGURE 5. Positive nuclear staining for MIB-1 in a recurrent maxillary HPC, proliferation index $=10.2 \%(\mathrm{H} \& \mathrm{E}$, original magnification $\times 40$ ). 
highest incidence is in the fifth and sixth decades. There is no sex predilection. ${ }^{2,3}$ An expanding mass that may be associated with pain is the common clinical presentation. ${ }^{3,8}$ Surgical excision is the preferred treatment, but local recurrence and metastasis can subsequently occur up to $50 \%$ of cases. ${ }^{2,4,9}$

Microscopically, HPC is characterized by a proliferation of pericytes in between variably sized, branching, thin-walled vascular channels. The pericytes have uniform, ovoid nuclei with indistinct cell borders and are located outside the reticulin sheath of the endothelium. Microscopic features ascribed prognostic value include increased cellularity, anaplasia, necrosis, hemorrhage, and prominent mitotic activity. ${ }^{3}$ Enzinger and Smith reported 8 of 16 HPCs that metastasized and had more than 4 mitoses per $10 \mathrm{hpf}$, whereas 46 of 66 cases without recurrence or metastasis had no mitotic figures in 30 hpf. The remaining 8 metastatic cases were not as mitotically active but were hypercellular, necrotic, or hemorrhagic. ${ }^{3}$ Similarly, McMaster, et $\mathrm{al}^{2}$ showed a general trend toward malignancy with hypercellular, mitotically active HPCs stratified into low, intermediate, and high-grade neoplasms.

Despite these features, it is difficult to predict the clinical outcome of HPC, including those in the head and neck. Although a trend toward malignancy is observed in intermediate and highgrade lesions, one study has shown that even lowgrade orbital HPCs have the potential for recurrence or metastasis. ${ }^{10}$

HPC of the head and neck can occur at any age. The more common sites specific in this region include the scalp, face, neck, oral cavity, nasal cavity, paranasal sinuses, and orbit. ${ }^{4,8,11}$ Treatment is primarily surgical, although some reports advocate the use of radiation therapy in those patients with a high-grade lesion or incomplete resections. ${ }^{12,13}$

HPCs of the head and neck are believed to behave more favorably compared with their counterparts in other anatomic sites. ${ }^{4,8,14}$ Walike and Bailey $^{8}$ reported a $44 \%$ local recurrence rate, but only 4 of 43 patients demonstrated metastasis. Although no apparent explanation for this less malignant behavior is evident, data from other reports show similar rates of recurrence and metastasis. ${ }^{4}$ Our data, although small in number, seem to support this observation with a recurrence rate of $25 \%$ and no mortalities.
Few reports are available on the immunohistochemical study of proliferation markers in HPC, and none specifically address their application to these tumors in the head and neck region. One report describes 7 of 9 recurrent or metastatic HPCs with proliferation rates of $15 \%$ or greater using an antibody to MIB-1. However, none of these cases originated in the head and neck. ${ }^{15} \mathrm{Yu}$ et $\mathrm{al}^{16}$ studied 25 HPCs of unspecified sites using a monoclonal antibody to proliferating cell nuclear antigen (PCNA). Patients with a PCNA staining of at least $14 \%$ experienced local or metastatic disease or have died, but those with staining less than $14 \%$ were all alive. As shown in Table 1, three recurrent cases had MIB-1 proliferative indices of $9.8 \%, 10.2 \%$, and $16.8 \%$. In contrast, most primary lesions possessed proliferation rates ranging from $2.6 \%$ to $4.5 \%$. Although the number of cases precludes statistical analysis, a proliferation index of $10 \%$ may serve as a useful predictor of a more aggressive biologic behavior. Of the 3 primary lesions with proliferation indices greater than 4.5\%, 2 have only 15 and 27 months of clinical follow-up, and the third is an infantile HPC.

Infantile or congenital HPC differs clinically and pathologically from the adult form. Congenital HPCs are predominantly subcutaneous head and neck lesions that display an indolent behavior despite worrisome histologic features such as extensive necrosis and hemorrhage, hypercellularity, and increased mitotic figures. ${ }^{17}$ Multiple congenital HPCs of the head and neck have been reported ${ }^{18}$ similar to our infantile case with more than 20 lesions that eventually regressed. This case exhibited hypercellularity, increased mitotic figures, and a proliferation rate of $19.6 \%$ in keeping with a tendency toward higher proliferation indices in congenital HPCs. ${ }^{16}$

In summary, HPC is an uncommon vascular tumor in which the biologic behavior is difficult to predict when based solely on conventional histologic parameters. Features suggestive of a highgrade lesion with an increased risk for subsequent recurrence or metastasis include increased cellularity, necrosis, hemorrhage, and increased mitotic activity. These features can be supplemented by determining the proliferation index using immunohistochemical techniques. A proliferation index of $10 \%$ or greater, as measured with MIB-1, may be indicative of a more aggressive subset of these rare neoplasms. 


\section{REFERENCES}

1. Stout A, Murray MR. Hemangiopericytomas: vascular tumors arising from Zimmerman's pericytes. Ann Surg 1942;116:26-33.

2. McMaster MJ, Soule EH, Ivins JC. Hemangiopericytoma: a clinicopathologic study and long-term followup of $60 \mathrm{pa}-$ tients. Cancer 1975;36:2232-2244.

3. Enzinger FM, Smith BH. Hemangiopericytoma: an analysis of 106 cases. Hum Pathol 1976;7:61-82.

4. Batsakis JG, Rice DH. The pathology of head and neck tumors: vasoformative tumors, part 9B. Head Neck 1981; 3:326-339.

5. Enzinger, FM, Weiss SW. Soft tissue tumors. St. Louis: Mosby-Year Book, Inc; 1995. 716 p.

6. Auguste L-J, Razack MS, Sako K. Hemangiopericytoma. J Surg Oncol 1982;20:260-264.

7. Pitluk HC, Conn J Jr. Hemangiopericytoma: literature review and clinical presentations. Am J Surg 1979;137: 413-416.

8. Walike JW, Bailey BJ. Head and neck hemangiopericytoma. Arch Otolaryngol 1971;93:345-353.

9. Gerner RE, Moore GE, Pickren JW. Hemangiopericytoma. Ann Surg 1974;179:128-132.

10. Croxatto JO, Font RL. Hemangiopericytoma of the orbit: A clinicopathologic study of 30 cases. Hum Pathol 1982; 13:210-218.
11. Carew JF, Singh B, Kraus DH. Hemangiopericytoma of the head and neck. Laryngoscope 1999;109:1409-1411.

12. Staples JJ, Robinson RA, Wen BC, Hussey DH. Hemangiopericytoma: the role of radiotherapy. Int J Radiat Oncol Biol Phys 1990;19:445-451.

13. Tran LM, Mark R, Meier R, Calcaterra TC, Parker RG. Sarcomas of the head and neck. Prognostic factors and treatment strategies. Cancer 1992;70:169-177.

14. Compagno J, Hyams VJ. Hemangiopericytoma-like intranasal tumors. A clinicopathologic study of 23 cases. Am J Clin Pathol 1976;66:672-683.

15. Middleton LP, Duray PH, Merino MJ. The histological spectrum of hemangiopericytoma: Application of immunohistochemical analysis including proliferative markers to facilitate diagnosis and predict prognosis. Hum Pathol 1998;29:636-640.

16. Yu CC-W, Hall PA, Fletcher CDM, et al. Haemangiopericytomas: the prognostic value of immunohistochemical staining with a monoclonal antibody to proliferating cell nuclear antigen (PCNA). Histopathology 1991;19:29-33.

17. Bailey PV, Weber TR, Tracy TF Jr, O'Connor DM, SoteloAvila C. Congenital hemangiopericytoma: An unusual vascular neoplasm of infancy. Surgery 1992;114:936-941.

18. Siebert JJ, Siebert RW, Weisenburger DS, Allsbrook W. Multiple congenital hemangiopericytomas of the head and neck. Laryngoscope 1978;88:1006-1012. 